\title{
Cooling rate dependence of the glass transition at free surfaces
}

\author{
S. Streit-Nierobisch, ${ }^{1, *}$ C. Gutt, ${ }^{2}$ M. Paulus, ${ }^{1}$ and M. Tolan ${ }^{1}$ \\ ${ }^{1}$ Fachbereich Physik, Technische Universität Dortmund, Otto-Hahn-Strasse 4, 44221 Dortmund, Germany \\ ${ }^{2}$ Deutsches Elektronen Synchrotron (HASYLAB), Notkestrasse 85, 22607 Hamburg, Germany
}

(Received 9 October 2007; published 29 January 2008)

\begin{abstract}
In situ x-ray reflectivity measurements are used to determine the cooling rate dependent freezing of capillary waves on the oligomer poly(propylene glycol). Only above the glass transition temperature $T_{\mathrm{G}}$ can the surface roughness $\sigma$ be described by the capillary wave model for simple liquids, whereas the surface fluctuations are frozen-in at temperatures below $T_{\mathrm{G}}$. As the state of a glass forming liquid strongly depends on its thermal history, this effect occurs for fast cooling rates already at a higher temperature than for slow cooling. For the fastest cooling rates a very large shift of $T_{\mathrm{G}}$ up to $240 \mathrm{~K}$ compared to the bulk value of $196 \mathrm{~K}$ was observed.

DOI: 10.1103/PhysRevB.77.041410

PACS number(s): 61.05.C-, 68.03.- g, 68.47.Mn
\end{abstract}

When a liquid is cooled towards the glass transition one observes a dramatic increase of its viscosity $\eta$ before the system forms a disordered glassy state. $\eta$ increases exponentially with decreasing temperature, and a most commonly used definition of the glass transition is the temperature where $\eta$ exceeds the value of $10^{12} \mathrm{~Pa} \mathrm{~s}$. As a result, in the bulk of glassy materials static frozen-in density fluctuations of long wavelengths exist. ${ }^{1}$ In thin films the glass transition temperature $T_{\mathrm{G}}$ changes as the film thickness is reduced, which strongly depends on the polymer-substrate interaction, ${ }^{2-6}$ e.g., a decrease in $T_{\mathrm{G}}$ is explained with an enhanced chain mobility near hard neutral walls (or air) due to a molten surface layer. ${ }^{5,7-9}$ Also a cooling rate dependence of $T_{\mathrm{G}}$ has been observed for thin films. ${ }^{10}$ However, as Jiang et al. have pointed out recently, viscoelastic effects for thin films differ significantly from bulk liquids, and the capillary wave model has to be modified to account for absorption effects at the substrate. ${ }^{11}$

At free surfaces and interfaces the additional boundary condition leads to hydrodynamic modes (i.e., capillary waves) which are present only near surfaces and interfaces. Capillary waves are generated by the thermal motion of the molecules in the liquid with the surface tension acting as restoring force of the perturbed surface. ${ }^{12}$ Therefore the amplitudes which correspond to the surface roughness are inversely proportional to the surface tension. The length scales $\Lambda$ of the capillary waves range from $10^{-2} \mathrm{~m}$ to the nanometer region and they are extending into the material underneath with a penetration depth of $2 \pi \Lambda$. The capillary waves lead to a surface roughness of most liquids on the order of $1-10 \AA$. As the relaxation time of the dynamics in the liquid is determined by the viscosity, for low viscosities propagating capillary waves occur on the surface, whereas for higher viscosities they are overdamped.

In this Rapid Communication we investigate if an analogous effect of freezing-in of fluctuations as known in the volume occurs at the free surface. We observed a strong cooling rate dependence of the glass transition at the free surface, which is not present in the bulk liquid.

Earlier x-ray scattering results on liquid glycerol have been hampered by the presence of a thin layer on top of the glycerol surface, ${ }^{13}$ and a previous study on the surface roughness on poly(propylene glycol) (PPG) showed no evidence of the glass transition because the covered temperature range was too small. ${ }^{14}$ We monitor now the freezing of the surface roughness of an oligomer depending on the applied cooling rate. A transition in the temperature dependence of the surface roughness to a partly frozen-in state is observed. The results are compared with the theory of the surface glass transition by Jäckle and Kawasaki. ${ }^{15,16}$

In order to characterize the surface glass transition, the height-height correlation function $C(R)=\langle z(r+R) z(r)\rangle_{r}$ with $z(r)$ denoting the vertical surface displacement from equilibrium at position $r$ is used. According to classical hydrodynamics, the structure factor, i.e., the Fourier transform of $C(R)$ of thermal height fluctuations on liquid surfaces is ${ }^{15}$

$$
S(q, T)=\frac{k_{\mathrm{B}} T}{\gamma(T)} \frac{1}{q^{2}+q_{\min }^{2}},
$$

with the Boltzmann constant $k_{\mathrm{B}}$, the absolute temperature $T$, the wave-vector transfer $q$, and the wave-vector cutoff $q_{\text {min }}$, which is given by gravity for cases of bulk liquids. $\gamma(T)$ denotes the temperature-dependent surface tension of the liquid. By integrating over the experimentally accessible $q$ range, the square of the surface roughness $\sigma$ can be calculated as ${ }^{15}$

$$
\sigma^{2}(T)=\frac{1}{2 \pi} \int_{q_{\min }}^{q_{\max }} S(q, T) q d q=\frac{k_{\mathrm{B}} T}{2 \pi \gamma(T)} \ln \left(\frac{q_{\max }}{q_{\min }}\right) .
$$

$q_{\max }$ is on the order of $2 \pi / d$ with the molecular diameter $d$. In an X-ray reflectivity experiment, $q_{\min }=q_{z} \Delta \alpha_{f} / 2$ is determined by the detector resolution $\Delta \alpha_{f}$ and depends on the wave-vector transfer perpendicular to the surface $q_{z}$ $=(4 \pi / \lambda) \sin \left(\alpha_{i}\right)$ with the wavelength $\lambda$ and the incident angle of the radiation with respect to the surface $\alpha_{i}$. Therefore in the liquid state the surface roughness depends on temperature via $\sigma^{2} \propto T / \gamma(T)$, which gives rise to a peculiar bending of $\sigma^{2}(T) .^{14}$

A supercooled liquid with increasing viscosity is discussed by Jäckle and Kawasaki as an incompressible medium with a Maxwell-Debye model for the frequency dependent viscosity $\eta(\omega)=\eta_{0} /(1-i \omega \tau) .{ }^{15,16} \quad \eta_{0}$ denotes the temperature-dependent low-frequency hydrodynamic value of the viscosity, and $\tau=\eta_{0}(T) \rho / G(\infty)$ is the relaxation time with the density $\rho$ and the high-frequency shear modulus 
$G(\infty)$. Below a certain temperature $T_{x}$, when $\tau$ exceeds the relaxation time of the shear modes in the liquid, the surface spectrum becomes more complex. Besides a modified quasielastic capillary wave spectrum $\tilde{S}(q, \omega)$, Rayleigh surface waves and a continuum of bulk elastic shear waves also occur. At even lower temperatures $T_{\mathrm{f}}$ the average shear stress relaxation time $\tau$ exceeds the experimental time scale and the quasielastic part of the fluctuation spectrum is frozen-in. Jäckle and Kawasaki identify $T_{\mathrm{f}}$ with the glass transition temperature. ${ }^{15,16}$

The structure factor of the "frozen" capillary wave fluctuations is calculated as follows: ${ }^{15}$

$$
S(q)_{\text {frozen }}=\frac{k_{\mathrm{B}} T_{\mathrm{f}}}{\gamma\left(T_{\mathrm{f}}\right)} \frac{1}{q^{2}\left[1+q l_{0}\left(T_{\mathrm{f}}\right) / 2\right]},
$$

with a length scale parameter $l_{0}=\gamma\left(T_{\mathrm{f}}\right) / G(\infty)$. The square of the surface roughness below $T_{\mathrm{f}}$ can then be expressed as a sum of a temperature-independent capillary wave spectrum and a temperature-dependent part,

$$
\sigma^{2}(T)=\frac{k_{\mathrm{B}} T_{\mathrm{f}}}{2 \pi \gamma\left(T_{\mathrm{f}}\right)} \ln \left(\frac{q_{\max }\left(l_{0} q_{\min }+2\right)}{q_{\min }\left(l_{0} q_{\max }+2\right)}\right)+\sigma^{\prime 2}(T),
$$

where $\sigma^{\prime 2}(T)=$ const $T$ accounts for the surface roughness due to Rayleigh surface waves and bulk elastic shear waves. This can be calculated by integrating the dynamic power spectrum of the thermal height fluctuations $S(q, \omega, T)$ (which is given in Ref. 15 for Rayleigh surface waves and bulk elastic shear waves) over all frequencies $\omega$ and then integrating over the accessible $q$ range according to Eq. (2).

In order to investigate the temperature dependence of the surface roughness given by Eq. (4), reflectivity measurements were performed in a wide temperature range with a Bruker D8-Advanced laboratory diffractometer in $\theta$ - $\theta$ geometry. The radiation is emitted from an $\mathrm{x}$-ray tube with a copper anode. Reflection from a Goebel mirror yields a parallel and monochromatic beam with a wavelength $\lambda=1.54 \AA$. The beam size on the sample is defined by a vertical slit of $0.1 \mathrm{~mm}$ height and a horizontal slit of $10 \mathrm{~mm}$ width. The scattered intensity is recorded by a scintillation detector. The detector slit height of $0.2 \mathrm{~mm}$ and the distance between the detector and the middle of the sample of $300 \mathrm{~mm}$ lead to an angular resolution $\Delta \alpha_{f}=0.7 \mathrm{mrad}$ in the scattering plane. Additional scans with a constant angular detector offset of $0.1^{\circ}$ were subtracted from the reflectivity scans to remove the diffuse background. A typical reflectivity measurement with offset scan was recorded within 6-8 h.

The investigated samples of PPG with an average molecular weight of $400 \mathrm{~g} / \mathrm{mol}$ were purchased from Alfa Aesar (product number 040811). According to capillary viscometer measurements, the viscosity $\eta$ of PPG-400 should follow the form $\ln \eta=-10.774+1047.3 \mathrm{~K} /(T-168.4 \mathrm{~K})$, therefore at room temperature the viscosity is $\eta=0.087 \mathrm{~Pa} \mathrm{~s} .{ }^{17}$ The surface tension $\gamma$ was measured by Dee and Sauer with the Wilhelmy method, leading to the expression $\gamma$ $=0.03431 \mathrm{~N} / \mathrm{m}-8 \times 10^{-5} \mathrm{~N} /(\mathrm{mK})(T-273.15 \mathrm{~K})$, which yields a surface tension of $0.033 \mathrm{~N} / \mathrm{m}$ at room temperature. ${ }^{18}$ Dielectric spectroscopy measurements for the bulk $T_{\mathrm{G}}$ value of PPG-400 result in $T_{\mathrm{G}}=196.4 \mathrm{~K} .{ }^{19}$ We performed differen- tial scanning calorimetry (DSC) measurements with a DSC-2 calorimeter from Perkin-Elmer. ${ }^{35}$ As for DSC measurements only a small fraction of around $18 \mathrm{mg}$ of the sample mass of several grams used for $\mathrm{x}$-ray reflectivity measurements can be inserted into the calorimeter, the resulting change in the heat flow-which is a marker for $T_{\mathrm{G}}$ - is too small at cooling rates slower than $20 \mathrm{~K} / \mathrm{min}$ to be measured. For the bulk value we obtained $T_{\mathrm{G}}=196.9 \mathrm{~K}$ for a cooling rate of $40 \mathrm{~K} / \mathrm{min}$ and $T_{\mathrm{G}}=197.1 \mathrm{~K}$ for $20 \mathrm{~K} / \mathrm{min}$ with an accuracy of $0.2 \mathrm{~K}$, which is in good agreement with Ref. 19. The $T_{\mathrm{G}}$ values nearly coincide within the error bars and can therefore only be estimated to exhibit a very weak dependence on the cooling rate.

The experimental setup used has been described in detail in Ref. 20. It is a two chamber design, where the outer cell is evacuated for thermal insulation, and the inner one (also evacuated to remove residual solvents and to minimize air scattering) contains a stainless steel trough $(120 \mathrm{~mm}$ diameter, $0.2 \mathrm{~mm}$ sample height) holding the sample. Homogeneous cooling is achieved by evaporating liquid nitrogen in a heat exchanger underneath the inner sample chamber with a constant flow rate, ensured by a control valve. The temperature is measured by two ceramic coated platinum resistor sensors (Pt100) dipped into the liquid sample. With this setup a temperature range from 185 to $300 \mathrm{~K}$ is covered with a temperature stability of $\pm 0.02 \mathrm{~K}$. After inserting the sample a waiting time of around $10 \mathrm{~h}$ is necessary to form a smooth surface. Then the sample is cooled at a constant rate with an accuracy of $\approx \pm 1 \mathrm{~K} / \mathrm{h}$ to the desired temperature, where the measurement is started as soon as $T$ is constant.

The x-ray reflectivity $R\left(q_{z}, T\right)$ of a liquid-vapor interface is given by ${ }^{21,22}$

$$
R\left(q_{z}, T\right)=R_{\mathrm{F}}\left(q_{z}\right) e^{-q_{z}^{2} \sigma^{2}\left(q_{z}, T\right)},
$$

where $R_{\mathrm{F}}$ is the Fresnel reflectivity of an ideally smooth surface. It should be noted here that in the exact expression for $R\left(q_{z}, T\right)$ an additional term, the so-called incomplete $\Gamma$ function $\Gamma[(1-\eta) / 2 ; A]$ with $A=2 \pi^{2} q_{\min }^{2} / q_{\max }^{2}$ and $\eta$ $=k_{\mathrm{B}} T q_{z}^{2} /(2 \pi \gamma)$ has to be multiplied on the right-hand side of Eq. (5). ${ }^{23}$ It is defined as $\Gamma(x, A)=\int_{A}^{\infty} t^{x-1} e^{-t} d t$ and arises from the integration of the scattering over all wave-vector transfers parallel to the surface $q_{x}{ }^{24}$ However, the $\Gamma$ function is near unity for small values of $\eta$, i.e., small $T$ and $q_{z}$, and can be neglected in this case: ${ }^{23}$ For PPG at the highest investigated temperature of $300 \mathrm{~K}$, the divergence in the $\Gamma$ function does not occur for $q_{z}<0.67 \AA^{-1}$, which is well above the accessible $q_{z}$ range with a laboratory diffractometer.

Typical reflectivities measured at the D8 laboratory diffractometer are plotted in Fig. 1 for eight different temperatures with fast cooling rates (for $T \lesssim 240 \mathrm{~K}$ ) ranging from around 15 to $20 \mathrm{~K} / \mathrm{h}$. The solid curves are fits using the Parratt algorithm ${ }^{25}$ where the effective roughness $\sigma(T)$ is independent of $q_{z}$. This is a good approximation for $\sigma$ because it depends only weakly on $q_{z}$. The only fit parameters are the dispersion $\delta$ and the roughness $\sigma$. The inset in Fig. 1 shows the $\ln R / R_{\mathrm{F}}$ vs $q_{z}^{2}$ plots of the same data.

$\sigma^{2}$ is plotted as a function of $T$ in Fig. 2 for all cooling rates. The solid line is a fit of $\sigma^{2}(T)$ for $T>240 \mathrm{~K}$ according 


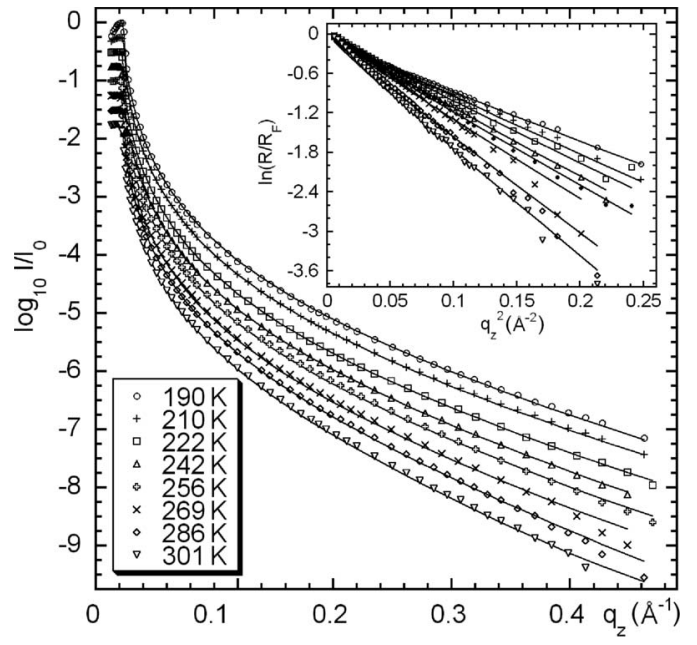

FIG. 1. Normalized intensity versus $q_{z}$ for fast cooling rates. The curves are shifted vertically for reasons of clarity. The inset shows the logarithm of the same data normalized by the Fresnel reflectivity as a function of $q_{z}^{2}$.

to Eq. (2) assuming a $T$ dependence of the surface tension $\gamma(T)=\gamma_{0}-A(T-273.15 \mathrm{~K})$ as predicted by Ref. 18 while the dashed lines represent the expression according to Eq. (4). Concerning the amount of data points at low temperatures it should be mentioned that each data point in Fig. 2 originates in $6-8 \mathrm{~h}$ of pure measurement time plus the time required for cooling the sample down at a certain rate, resulting in $\approx 24 \mathrm{~h}$ per data point. Therefore only a limited number of data points could be acquired in a reasonable time range. For low cooling rates the surface roughness follows a liquidlike behavior until close to the bulk $T_{\mathrm{G}}$ value of $196 \mathrm{~K}$. This is in good agreement with previous findings from Ref. 14. However, with increasing cooling rates the $T$ dependence of the surface roughness changes. Below a temperature $T_{\mathrm{f}}$ the

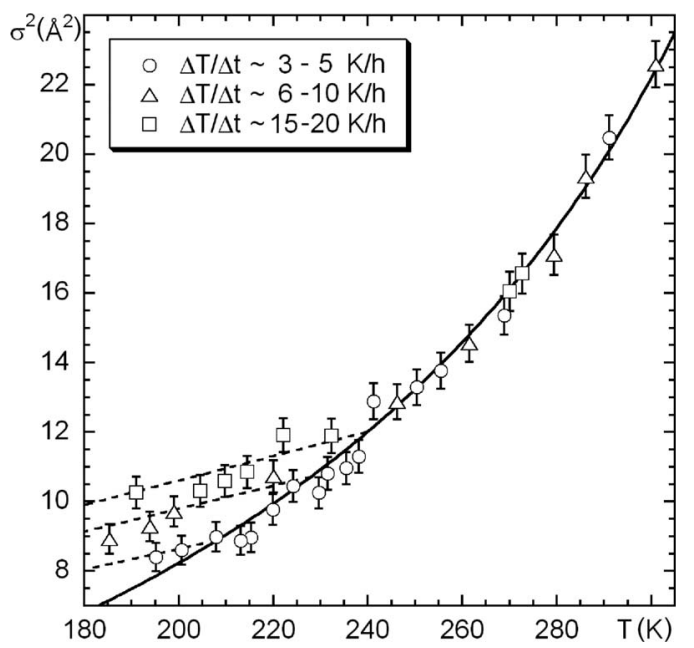

FIG. 2. Square of the surface roughness vs temperature for fast (15-20 K/h, squares), medium $(6-10 \mathrm{~K} / \mathrm{h}$, triangles), and slow (3-5 K/h, circles) cooling rates. The solid line represents a fit according to Eq. (2) for $T>240 \mathrm{~K}$ and is extrapolated to smaller temperatures. The dashed lines are calculations according to Eq. (4) with the adjusted length scale parameter $l_{0}=(2000 \pm 1000) \AA$. roughness for high cooling rates is larger than predicted by capillary wave theory. At this temperature the surface fluctuations are falling out of equilibrium and parts of them are frozen-in. It is of special interest that the surface roughness below $T_{\mathrm{f}}$ still depends on $T$, i.e., the fluctuations do not freeze completely, even for very high cooling rates. This is in good agreement with the predictions from Jäckle et al. [Eq. (4)] which are presented as dashed lines in Fig. 2. We therefore identify $T_{\mathrm{f}}$ as the glass transition temperature $T_{\mathrm{G}}$.

The transition temperature where the surface fluctuations freeze partially in is estimated from Fig. 2. For slow cooling rates the frozen-in state is reached at $T \approx 208 \mathrm{~K}$, whereas at faster cooling rates the transition occurs already at higher temperatures $(T \approx 228 \mathrm{~K}$ for medium and $240 \mathrm{~K}$ for fast cooling). The error in the determination of the transition temperature is estimated as $\pm 5 \mathrm{~K}$. The required cooling rate for reaching the equilibrium value, i.e., for the surface to stay fluid down to the bulk $T_{\mathrm{G}}$ value, should at least be smaller than $3 \mathrm{~K} / \mathrm{h}$. For the slowest cooling rate it should be noted that due to the limited amount of data points $T_{\mathrm{G}_{0}}$ is not well defined. Also the error for $l_{0}=(2000 \pm 1000) \AA$ is rather large, because a small deviation in the slope of the dashed lines results in a large change of $l_{0}$. According to Ref. $15, l_{0}$ should be on the order of 1-10 $\AA$. However, x-ray photon correlation spectroscopy measurements on PPG (not yet published) result in similar high values of $l_{0}$ of several thousand $\AA$, indicating that $G(\infty)$ may indeed be about a factor 1000 smaller, i.e., on the order of $10^{5} \mathrm{~Pa}$, than expected from rheological measurements. It is still a current topic of research if the Maxwell-Debye model used in Ref. 15 is valid or might have to be modified in order to explain the small values of $G(\infty) .{ }^{26}$ As capillary waves display low frequencies close to $T_{\mathrm{G}}, G(\infty)$ probably overestimates the value of the shear modulus relevant for the low frequencies of the surface fluctuations close to $T_{\mathrm{G}}$.

Although the data points in Fig. 2 for $T>240 \mathrm{~K}$ are obtained with different cooling rates, one single fit curve is able to describe the temperature dependence, because for $T$ $\gtrsim 240 \mathrm{~K}$ the relaxation is fast enough that no change in the roughness due to different cooling rates can be detected within the error bars. To our knowledge, this strong dependence of the glass transition temperature on the cooling rate has not been observed at the surface before. $T_{\mathrm{G}}$ changes in a broad range, reaching up to $240 \mathrm{~K}$ for the fastest cooling rate compared to $196.4 \mathrm{~K}$ in the bulk. ${ }^{19}$ In the bulk, the dependence of $T_{\mathrm{G}}$ on the cooling rate is typically rather weak, as a change in the cooling rate by an order of magnitude causes a change of $T_{\mathrm{G}}$ by $3-5 \mathrm{~K} \cdot{ }^{27,28}$ Mode coupling theory calculations predicted a change of $T_{\mathrm{G}}$ of $5 \mathrm{~K}$ per decade of the cooling rate, ${ }^{29}$ which is in good agreement with experiments, e.g., for $\mathrm{B}_{2} \mathrm{O}_{3}$ in Ref. 30. Our DSC measurements confirm that $T_{\mathrm{G}}$ in the bulk occurs at around $197 \mathrm{~K}$, nearly independent of the applied cooling rate. Although the calorimeter cannot match the slow cooling rates of our surface experiments, the obtained results are nevertheless comparable due to the following reasons: Regarding the nature of the glass transition and its cooling rate dependence it does not seem realistic to expect a stronger effect on $T_{\mathrm{G}}$ in the bulk by lowering the cooling rate. Thus, the DSC scans provide a lower limit for the effect we observed, i.e., with lower cool- 
ing rates the bulk $T_{\mathrm{G}}$ would even shift to lower values and the effect we observed would be even stronger.

We also investigated whether a subsequent freezing-in of the capillary wave spectrum can explain the observed temperature dependence of the roughness: A typical reflectivity scan takes several hours, therefore capillary waves with even longer relaxation times may appear as frozen-in with regards to the surface roughness. With decreasing $T$ the surface roughness can then be iteratively calculated by a sum of a frozen-in part and a still fluctuating part by using the bulk values of the viscosity and the surface tension. The square of the surface roughness then follows in analogy to the left part of Eq. (2), with the difference that the integral is subdivided into two parts which are summed up. The first integral runs from $q_{\min }$ to $q^{\prime}$ and the second one from $q^{\prime}$ to $q_{\max }$ with $q^{\prime}$ $=[1 / \tau][2 \eta(T) / \gamma(T)]$ as calculated in Refs. 31 and 32. The surface roughness calculated in this way, however, is identical to the solid line in Fig. 2 and shows a freezing-in of the surface roughness at temperatures close to the bulk $T_{\mathrm{G}}$ only.

It should also be mentioned that other methods such as single molecule fluorescence spectroscopy to determine $T_{\mathrm{G}}$ in confined liquids, finding a more mobile surface layer, ${ }^{33}$ probe different physical properties of the surface and add to the complications of the surface glass transition. A more mobile surface layer would result in a lower viscosity which may be probed with $\mathrm{x}$-ray photon correlation spectroscopy (XPCS) measurements. Capillary waves are connected with long-range correlations with a penetration depth proportional to $1 / q$. The static average of the capillary waves over all $q$ values corresponds to the surface roughness. The freezing-in of long length scale capillary waves, however, does not inherently imply that motions on molecular length scales are frozen in. The existence of a more mobile surface layer probed by XPCS has been discussed in Ref. 34 .

In summary, we were able to observe the freezing-in of the surface roughness for a free surface. We found an anomalous strong dependence of the surface glass transition temperature on the cooling rate which is not present in the bulk liquid.

This work was supported by the Federal Ministry for Education and Research (Contract No. BMBF 05 KS4PEA/3). We would like to acknowledge the help of H. Schulte and H.-L. Keller from the Inorganic Chemistry Department of the University Dortmund for performing the DSC scans.
*Present address: Deutsches Elektronen Synchrotron (HASYLAB), Notkestr. 85, 22607 Hamburg, Germany; simone.streitnierobisch@desy.de

${ }^{1}$ N. Laberge, V. Vasilescu, C. Montrose, and P. Macedo, J. Am. Ceram. Soc. 56, 506 (1973).

${ }^{2}$ J. L. Keddie, R. A. L. Jones, and R. A. Cory, Europhys. Lett. 27, 59 (1994).

${ }^{3}$ W. E. Wallace, J. H. van Zanten, and W. L. Wu, Phys. Rev. E 52, R3329 (1995); J. H. van Zanten, W. E. Wallace, and W. L. Wu, ibid. 53, R2053 (1996).

${ }^{4}$ J. A. Forrest, K. Dalnoki-Veress, J. R. Stevens, and J. R. Dutcher, Phys. Rev. Lett. 77, 2002 (1996); 77, 4108 (1996).

${ }^{5}$ J. A. Forrest, K. Dalnoki-Veress, and J. R. Dutcher, Phys. Rev. E 56, 5705 (1997).

${ }^{6}$ J. S. Sharp and J. A. Forrest, Phys. Rev. Lett. 91, 235701 (2003).

${ }^{7}$ J. A. Forrest and J. Mattsson, Phys. Rev. E 61, R53 (2000).

${ }^{8}$ S. Herminghaus, R. Seemann, and K. Landfester, Phys. Rev. Lett. 93, 017801 (2004).

${ }^{9}$ F. L. Pratt, T. Lancaster, M. L. Brooks, S. J. Blundell, T. Prokscha, E. Morenzoni, A. Suter, H. Luetkens, R. Khasanov, R. Scheuermann, U. Zimmermann, K. Shinotsuka, and H. E. Assender, Phys. Rev. B 72, 121401(R) (2005).

${ }^{10}$ Z. Fakhraai and J. A. Forrest, Phys. Rev. Lett. 95, 025701 (2005).

${ }^{11}$ Z. Jiang, H. Kim, X. Jiao, H. Lee, Y.-J. Lee, Y. Byun, S. Song, D. Eom, C. Li, M. H. Rafailovich, L. B. Lurio, and S. K. Sinha, Phys. Rev. Lett. 98, 227801 (2007).

${ }^{12}$ R. Loudon, Proc. R. Soc. London, Ser. A 372, 275 (1980).

${ }^{13}$ T. Seydel, M. Tolan, B. M. Ocko, O. H. Seeck, R. Weber, E. DiMasi, and W. Press, Phys. Rev. B 65, 184207 (2002).

${ }^{14}$ M. Sprung, T. Seydel, C. Gutt, R. Weber, E. DiMasi, A. Madsen, and M. Tolan, Phys. Rev. E 70, 051809 (2004).

${ }^{15}$ J. Jäckle and K. Kawasaki, J. Phys.: Condens. Matter 7, 4351 (1995).

${ }^{16}$ J. Jäckle, J. Phys.: Condens. Matter 10, 7121 (1998).
${ }^{17}$ J. Cochrane, G. Harrison, J. Lamb, and D. W. Phillips, Polymer 21, 837 (1980).

${ }^{18}$ G. T. Dee and B. B. Sauer, J. Colloid Interface Sci. 152, 85 (1992).

${ }^{19}$ J. Schüller, Yu. B. Mel'nichenko, R. Richert, and E. W. Fischer, Phys. Rev. Lett. 73, 2224 (1994).

${ }^{20}$ T. Seydel, A. Madsen, M. Sprung, M. Tolan, G. Grübel, and W. Press, Rev. Sci. Instrum. 74, 4033 (2003).

${ }^{21}$ A. Braslau, M. Deutsch, P. S. Pershan, A. H. Weiss, J. AlsNielsen, and J. Bohr, Phys. Rev. Lett. 54, 114 (1985).

${ }^{22}$ A. Braslau, P. S. Pershan, G. Swislow, B. M. Ocko, and J. AlsNielsen, Phys. Rev. A 38, 2457 (1988).

${ }^{23}$ T. Ghaderi, Ph.D. thesis, University of Dortmund, 2006.

${ }^{24}$ M. K. Sanyal, S. K. Sinha, K. G. Huang, and B. M. Ocko, Phys. Rev. Lett. 66, 628 (1991).

${ }^{25}$ L. G. Parratt, Phys. Rev. 95, 359 (1954).

${ }^{26}$ H. Sternemann, Diploma thesis, University of Dortmund, 2005.

${ }^{27}$ P. G. Debenedetti and F. H. Stillinger, Nature (London) 410, 259 (2001).

${ }^{28}$ M. D. Ediger, C. A. Angell, and S. R. Nagel, J. Phys. Chem. 100, 13200 (1996).

${ }^{29}$ K. Vollmayr, W. Kob, and K. Binder, Phys. Rev. B 54, 15808 (1996).

${ }^{30}$ R. Brüning and M. Sutton, Phys. Rev. B 49, 3124 (1994).

${ }^{31}$ V. G. Levich, Physicochemical Hydrodynamics (Prentice-Hall, Englewood Cliffs, NJ, 1962).

${ }^{32}$ M.-A. Bouchiat and J. Meunier, J. Phys. (France) 32, 561 (1971).

${ }^{33}$ C. J. Ellison and J. M. Torkelson, Nat. Mater. 2, 695 (2003).

${ }^{34}$ Z. Jiang, H. Kim, S. G. J. Mochrie, L. B. Lurio, and S. K. Sinha, Phys. Rev. E 74, 011603 (2006).

${ }^{35}$ See EPAPS Document No. E-PRBMDO-77-R15804 for differential scanning calorimetry measurements. For more information on EPAPS, see http://www.aip.org/pubservs/epaps.html. 